\title{
VARIETIES OF NILPOTENT GROUPS OF CLASS FOUR (III)
}

\section{PATRICK FITZPATRICK}

(Received 5 June 1981)

Communicated by D. E. Taylor

\begin{abstract}
In this paper we complete the investigation of those varieties of nilpotent groups of class (at most) four whose free groups have no nontrivial elements of odd order. Each such variety is labelled by a vector of sixteen parameters, each parameter a nonnegative integer or $\infty$, subject to numerous but simple conditions. Each vector satisfying these conditions is in fact used and directly yields a defining set of laws for the variety it labels. Moreover, one can easily recognise from the parameters whether one variety is contained in another. In view of the reduction carried out in the first paper of this series (written jointly with L. G. Kovács) this completes the determination of all varieties of nilpotent groups of class four.
\end{abstract}

1980 Mathematics subject classification (Amer. Math. Soc.): 20 E 10.

\section{Introduction}

This paper completes the determination of all varieties of nilpotent groups of class at most four begun in Fitzpatrick and Kovács (1983) and continued in Fitzpatrick (1983); these will be referred to as I and II respectively. The results of the latter will be used so extensively in the present work that it is convenient to adopt the following convention: (a.b) refers to the result numbered a.b in II. In addition we now adopt in toto the notation of that paper.

The outstanding problem is to determine those $2^{\prime}$-torsionfree subvarieties of $\mathfrak{R}_{4}$ which do not contain $\mathfrak{R}_{3}$ or, equivalently, those $2^{\prime}$-isolated fully invariant subgroups of the free group $F$ of rank 4 of $\Re_{4}$ not contained in $N_{3}=\Re_{3}(F)$. This is achieved in three steps. First those lying in $N_{2}=\mathfrak{R}_{2}(F)$, then those contained in $F^{\prime}$ and finally those not contained in $F^{\prime}$ are found: details appear in Sections 3,

c Copyright Australian Mathematical Society 1983 
4 and 5 respectively. In Section 2 we show how the methods of II can be adapted to yield the solutions for the first two of these steps.

\section{Preliminary lemmas}

The first two results are based on the well-known classification of varieties of nilpotent groups of class at most three (see, for example, Jónsson (1966)).

2.1 Lemma. The 2'-isolated fully invariant subgroups $H_{1}$ of $F$ such that $N_{3}<H_{1}$ $\leqslant F^{\prime}$ but $H_{1} \neq N_{2}$, are in one-to-one correspondence with the ordered pairs $(r, s)$ of nonnegative integers such that $r \geqslant s$, the subgroup corresponding to this pair being $\mathfrak{B}_{2^{r}}\left(F^{\prime}\right) N_{2}^{2^{s}} N_{3}$.

2.2 LEMMA. The 2'-isolated fully invariant subgroups $\mathrm{H}_{2}$ of $\mathrm{F}$ such that $\mathrm{N}_{3}<\mathrm{H}_{2}$ $\leqslant N_{2}$ are in one-to-one correspondence with the nonnegative integers $s$, the subgroup corresponding to s being $\mathrm{N}_{2}^{2^{s}} \mathrm{~N}_{3}$.

Here we have written $N_{2}^{2^{s}}$ for the subgroup $\left\{w^{2^{s}} \mid w \in N_{2}\right\}$, a convention we shall employ with any abelian group in place of $N_{2}$. We shall also use frequently, and without any further reference, the result of I that products of 2 '-isolated fully invariant subgroups of $F$ are 2 -isolated. Simple commutator calculations show that in $F$

$$
\left[[x, y]^{2^{r}}, z\right]=[x, y, z]^{2^{r}} \text { and }\left[[x, y, z]^{2^{s}}, t\right]=[x, y, z, t]^{2^{s}}
$$

so we have that

$$
\left[\mathfrak{B}_{2^{r}}\left(F^{\prime}\right) N_{2}^{2^{s}} N_{3}, F\right]=N_{2}^{2^{r}}{N_{3}^{2^{s}}}^{2^{\prime}} \text { and }\left[N_{2}^{2^{s}}, F\right]=N_{3}^{2^{s}} .
$$

2.4 LemMa. If two endomorphisms of $F$ agree on $F / F^{\prime}$, they also agree on $\mathfrak{B}_{2^{r}}\left(F^{\prime}\right) N_{2}^{2^{s}} N_{3} / N_{2}^{2^{r}} N_{3}^{2^{s}}$.

Proof. Let $\varphi, \psi$ be endomorphisms of $F$ which agree on $F / F^{\prime}$. As is well known, $\varphi$ and $\psi$ then agree on $F^{\prime} / N_{2}$, on $N_{2} / N_{3}$ and on $N_{3}$. In particular, if $a \in F^{\prime}$ then $a \varphi=(a \psi) w$ for some $w \in N_{2}$. Since $N_{2}$ is central in $F^{\prime}$, it follows that $a^{2^{r}} \varphi=(a \varphi)^{2^{r}}=(a \psi)^{2^{r}} w^{2^{r}}=\left(a^{2^{r}} \psi\right) w^{2^{r}}$; thus $\varphi$ and $\psi$ agree on the element $a^{2^{r}} N_{2}^{2^{r}} N_{3}^{2^{s}}$ of our quotient. By a similar argument, they also agree on the elements $b^{2^{s}} N_{2}^{2^{r}} N_{3}^{2^{s}}$ with $b \in N_{2}$ and $c N_{2}^{2^{r}} N_{3}^{2^{s}}$ with $c \in N_{3}$. The quotient is generated by such elements (as group, not only as fully invariant subgroup), so it follows that $\varphi$ and $\psi$ agree on it. 
Similarly,

2.5 LeMMA. If two endomorphisms of $F$ agree on $F / F^{\prime}$, they also agree on $N_{2}^{2^{s}} N_{3} / N_{3}^{2^{s}}$.

Suppose now that $H$ is any $2^{\prime}$-isolated fully invariant subgroup of $F$ contained in $F^{\prime}$. If $H \leqslant N_{3}$ then $H$ has been dealt with in II, so suppose $H \neq N_{3}$. We have to look at two cases separately.

First, we may have $H \leqslant N_{2}$. Then by 2.2 there is a unique nonnegative integer $s$ such that $\mathrm{HN}_{3}=\mathrm{N}_{2}^{2^{s}} \mathrm{~N}_{3}$. All $\mathrm{H}$ which correspond to the same $s$ lie between $\mathrm{N}_{2}^{2^{s}} N_{3}$ and $N_{3}^{2^{s}}$, for $H \geqslant[H, F]=\left[H N_{3}, F\right]=N_{3}^{2^{s}}$ because $N_{3}$ is central in $F$ and 2.3 applies. Thus the study of these $H$ is equivalent to the investigation of the $2^{\prime}$-isolated (End $F$ )-submodules of $N_{2}^{2^{s}} N_{3} / N_{3}^{2^{s}}$. We know from 2.5 that this ( $2^{\prime}$-torsionfree, central) section of $F$ may as well be viewed as an (End $F / F^{\prime}$ )module, that is, as $G$-module where $G=\operatorname{Mat}^{\times}(4, Z)$ in accordance with the notation of II. Hence the methods of that paper can handle the problem.

Second, suppose $H \$ N_{2}$. A similar argument using 2.1, 2.3 and 2.4 shows that the study of these subgroups $H$ is equivalent to the investigation of the $2^{\prime}$-isolated submodules of the (2'-torsionfree, central) sections $B_{2^{r}}\left(F^{\prime}\right) N_{2}^{2^{s}} N_{3} / N_{2}^{2^{r}} N_{3}^{2^{s}}$ of $F$ regarded as $G$-modules. Thus our previous methods can also cope with this case.

Before proceeding to give the details it is convenient at this stage to record the results of some elementary commutator calculations in $F$. Recall that $F$ is freely generated by $\{x, y, z, t\}$.

2.6 Lemma. Let $a, b, c \in\{x, y, z, t\}$. Then for each $\varphi \in G,[a, b, c]^{2^{s}} \varphi$ is congruent modulo $N_{3}^{2^{s}}$ to a product $\Pi_{i} w_{i}^{n(i)}$ where $w_{i}=\left[a_{i}, b_{i}, c_{i}\right]^{2^{s}}$ with $a_{i}, b_{i}, c_{i} \in$ $\{x, y, z, t\}$ and the $n(i)$ are integers.

PRoof. Since $N_{3}$ is central the element $[a, b, c]^{2^{s}} \varphi$ can be expressed as a product $u^{2^{s}} v^{2^{s}}$ where $u$ is a product of commutators $\left[a_{i}, b_{i}, c_{i}\right]$ and $v \in N_{3}$. Thus modulo $N_{3}^{2^{s}}, v^{2^{s}}$ is trivial and since $N_{2}$ itself is central $u^{2^{s}}$ can be split up as a product of the required form.

2.7 Lemma. Let $a, b \in\{x, y, z, t\}$. Then for each $\varphi \in G,[a, b]^{2^{r}} \varphi$ is congruent modulo $N_{2}^{2^{2}} N_{3}^{2^{s}}$ to a product $\Pi_{i} w_{i}^{n(i)}$ where either $w_{i}=\left[a_{i}, b_{i}\right]^{2^{r}}$ or $w_{i}=$ $\left[a_{i}, b_{i}, c_{i}, d_{i}\right]^{2^{-1}}$ with $a_{i}, b_{i}, c_{i}, d_{i} \in\{x, y, z, t\}$ and the $n(i)$ are integers.

Proof. In this case since $N_{2}$ is central the element $[a, b]^{2^{r}} \varphi$ can be expressed as a product $u^{2^{r}} v^{2^{r}}$ where $u$ is a product of commutators $\left[a_{i}, b_{i}\right]$ and $v \in N_{2}$. Thus 
modulo $N_{2}^{2^{r}} N_{3}^{2^{s}}, v^{2^{r}}$ is trivial. Since $F^{\prime}$ has class two we have

$$
\left(\left[a_{1}, b_{1}\right]\left[a_{2}, b_{2}\right]\right)^{2^{r}}=\left[a_{1}, b_{1}\right]^{2^{r}}\left[a_{2}, b_{2}\right]^{2^{r}}\left[a_{2}, b_{2}, a_{1}, b_{1}\right]^{2^{r-1}\left(2^{r}-1\right)} .
$$

When there are more than two factors in $u$ the pattern is similar.

\section{Subgroups of $N_{2}$}

The abelian group $N_{2}^{2^{s}} N_{3} / N_{3}^{2^{s}}$ is a direct sum of 20 infinite cyclic groups and 60 cyclic groups of order $2^{s}$ generated respectively by cosets with representatives the $2^{s}$-powers of the basic commutators of weight three and by the basic commutators of weight four. Let $X$ stand for the tensor product of this $G$-module (over $Z$ ) with $Z_{(2)}$ regarded as $Z_{(2)} G$-module in the obvious way. We shall temporarily use group commutators as labels for the corresponding elements of $X$ : thus the basic commutators just mentioned are regarded as generators of the corresponding cyclic direct summands of $X$. Section 3 of II may now be applied to reduce the study of submodules of $X$ to that of the $\varepsilon Z_{(2)} G \varepsilon$-submodules of $X \varepsilon$. Observe that $\varepsilon_{1}$ annihilates $X$ so that $X=X \varepsilon_{2} \oplus X \varepsilon_{3} \oplus X \varepsilon_{4}$. Besides the 18 generators corresponding to commutators of weight four which are fixed by $\varepsilon$ there are precisely 4 of weight three namely

$$
[y, x, x]^{2^{s}}, \quad[y, x, y]^{2^{s}}, \quad[y, x, z]^{2^{s}} \text { and }[z, x, y]^{2^{s}} .
$$

These 22 form a generating set for $X \varepsilon$. As before however it is convenient to change to a different generating set.

Recall that in Section 4 of II certain elements of $W \varepsilon$ were designated $\mathbf{a}_{1}, \ldots, \mathbf{a}_{18}$. We shall now use the same symbols to represent the corresponding elements of $X \varepsilon$. Thus $\mathbf{a}_{1}$ denotes the image in $X$ of the coset of $N_{2}^{2} N_{3} / N_{3}^{2^{4}}$ represented by the group commutator $[y, x, x, y][y, x, x, x][x, y, y, y]^{-1}$. In addition we define in the same way

$$
\begin{array}{ll}
\mathbf{x}_{1}=[y, x, x]^{2^{s}}, & \mathbf{x}_{3}=[z, y, x]^{2^{s}}, \\
\mathbf{x}_{2}=[x, y, y]^{2^{s}}, & \mathbf{x}_{4}=[z, x, y]^{2^{s}} .
\end{array}
$$

We then have

3.1 LEMMA. The following are generating sets for the $X \varepsilon_{i}$ :

$$
\begin{aligned}
& X \varepsilon_{2}:\left\{\mathbf{x}_{1}, \mathbf{x}_{2}, \mathbf{a}_{1}, \mathbf{a}_{2}, \mathbf{a}_{3}\right\}, \\
& X \varepsilon_{3}:\left\{\mathbf{x}_{3}, \mathbf{x}_{4}, \mathbf{a}_{4}, \ldots, \mathbf{a}_{12}\right\}, \\
& X \varepsilon_{4}:\left\{\mathbf{a}_{13}, \ldots, \mathbf{a}_{18}\right\} .
\end{aligned}
$$


Proof. This follows directly from (4.2) and the following equations which hold in $X$ :

$$
\begin{aligned}
& {[x, y, y]^{2^{s}}=-[y, x, y]^{2^{s}},} \\
& {[z, y, x]^{2^{s}}=-[y, x, z]^{2^{s}}+[z, x, y]^{2^{s}} .}
\end{aligned}
$$

With reference to the generating set $\mathbf{x}_{1}, \ldots, \mathbf{x}_{4}, \mathbf{a}_{1}, \ldots, \mathbf{a}_{18}$ we can identify End $_{Z_{(2)}} X \varepsilon$ with a certain homomorphic image of Mat $\left(22, Z_{(2)}\right)$ according to a well known formula (see, for example, Jacobson (1974) Section 3.11). Rather than introduce cumbersome notation which would be used only briefly we shall avoid describing that matrix algebra explicitly preferring instead simply to use information about it in what follows. Let $S_{1}$ stand for the image of $\varepsilon Z_{(2)} G \varepsilon$ in $\operatorname{End}_{Z_{(2)}} X \varepsilon$. We obtain a generating set for $S_{1}$ from (3.4) and our aim is to write down the matrices representing this generating set.

Now the lower left $18 \times 4$ submatrix of each element of End $_{Z_{(2)}} X \varepsilon$ is the zero matrix and by virtue of 2.6 the upper right $4 \times 18$ submatrix of each element of $S_{1}$ is also the zero matrix. Thus each matrix in $S_{1}$ is the direct sum of a $4 \times 4$ and an $18 \times 18$ matrix. For the generators of $S_{1}$ the $18 \times 18$ direct summands can be obtained simply by reading the corresponding matrix of $S$ (given in Section 4 of II) modulo $2^{s} Z_{(2)}$. It remains to give the $4 \times 4$ parts. Note that we are transferring the labels of the elements of $S$ wholesale to the corresponding elements of $S_{1}$.

3.2 Action on $\mathrm{X \varepsilon}_{2}$.

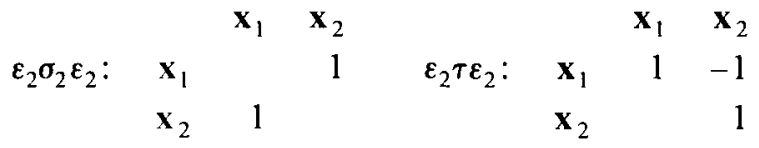

$$
\begin{aligned}
& \begin{array}{llllllrr} 
& & \mathbf{x}_{1} & \mathbf{x}_{2} & & & \mathbf{x}_{3} & \mathbf{x}_{4} \\
\varepsilon_{2} \mu_{\kappa} \varepsilon_{2}: & \mathbf{x}_{1} & \kappa^{2} & & (2 \rightarrow 3): & \mathbf{x}_{1} & 1 & 1 \\
\mathbf{x}_{2} & & \kappa & & \mathbf{x}_{2} & &
\end{array}
\end{aligned}
$$

3.3. Action on $X \varepsilon_{3}$.

$$
\begin{aligned}
& \begin{array}{rrrrrrrr}
\varepsilon_{3} \sigma_{2} \varepsilon_{3}: & & \mathbf{x}_{3} & \mathbf{x}_{4} & & & \mathbf{x}_{3} & \mathbf{x}_{4} \\
& \mathbf{x}_{3} & 1 & \varepsilon_{3} \sigma_{3} \varepsilon_{3}: & \mathbf{x}_{3} & & -1 \\
\mathbf{x}_{4} & 1 & & & \mathbf{x}_{4} & 1 & -1
\end{array}
\end{aligned}
$$

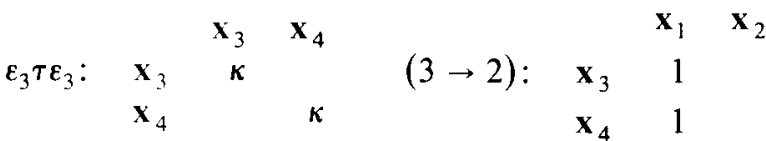

Our investigation of $S_{1}$ follows closely the pattern established in Section 4 of II. Referring to the elements $\xi_{1}, \ldots, \xi_{12}$ of $S_{1}$ we find by direct calculation that for 
$i \notin\{2,3\}, \mathbf{x}_{1} \xi_{i}=\mathbf{x}_{2} \xi_{i}=0$ while $\xi_{2}$ and $\xi_{3}$ act on $X \varepsilon_{2}$ as $\operatorname{diag}(1,0,0,1,0)$ and $\operatorname{diag}(0,1,0,0,1)$ respectively. Action on $X \varepsilon_{3}$ is not quite as straightforward: for $i \notin\{4,5\}, \mathbf{x}_{3} \xi_{i}=\mathbf{x}_{4} \xi_{i}=0$ but $\xi_{4}, \xi_{5}$ are not idempotent as they stand. Define

$$
\nu=\left(\xi_{4}+\xi_{5}\right) \varepsilon_{3} \llbracket \sigma_{3}-3\left(\mu_{-1}+1\right)-(\tau-1) \sigma_{2} \rrbracket \varepsilon_{3}\left(\xi_{4}+\xi_{5}\right)
$$

with bracket notation as in II. Then

$$
\hat{\xi}_{4}=\frac{1}{3} \xi \llbracket 1-\sigma_{3}+\nu \sigma_{3}-\nu \sigma_{3}^{2} \rrbracket \xi
$$

and

$$
\hat{\xi}_{5}=\frac{1}{3} \xi \llbracket 1-\sigma_{3}^{2}-\nu \sigma_{3}+\nu \sigma_{3}^{2} \rrbracket \xi
$$

act on $X \varepsilon_{3}$ as $\operatorname{diag}(1,0,1,0, \ldots, 0)$ and $\operatorname{diag}(0,1,0,1,0, \ldots, 0)$ respectively. We have thus proved the first of the following lemmas.

3.4 Lemma. The $\xi_{1}, \ldots, \xi_{3}, \hat{\xi}_{4}, \hat{\xi}_{5}, \xi_{6}, \ldots, \xi_{12}$ are pairwise orthogonal idempotents with sum 1 in $S_{1}$.

3.5 LeMma. The following elements are in $S_{1}$ :

$$
\begin{array}{ll}
e_{22}(i, j)+e_{22}(i+5, j+5), & i, j \in\{1,2,3,4\}, \\
e_{22}(i, j)+e_{22}(i+1, j+1), & i, j \in\{11,13,15,17,19\} .
\end{array}
$$

Proof. This adapted from (4.7). The $e_{18}(i, j)+e_{18}(i+1, j+1)$ given there translate directly to the $e_{22}(i, j)+e_{22}(i+1, j+1)$. The $e_{18}(i, j)$ for $i, j \in\{2,3\}$ or $i, j \in\{4,5\}$ also translate directly to give the corresponding $e_{22}(i, j)+$ $e_{22}(i+5, j+5)$. For the others a minor modification is necessary: we obtain $e_{22}(1,3)+e_{22}(6,8)$ using $\xi_{2}(2 \rightarrow 3)[2-\nu] \hat{\xi}_{4}$ and $e_{22}(1,4)+e_{22}(6,9)$ using $\xi_{2}(2 \rightarrow 3)[2 \nu-1] \hat{\xi}_{5}$. The other $e_{22}(i, j)+e_{22}[i+5, j+5]$ are found from these by multiplication so the lemma is proved.

\section{Rename}

$$
\begin{aligned}
& e_{22}(5,5)=\varepsilon_{11}^{1}, \\
& e_{22}(i, j)+e_{22}(i+5, j+5)=\varepsilon_{i j}^{2}, \quad i, j \in\{1,2,3,4\}, \\
& e_{22}(10,10)=\varepsilon_{11}^{3}, \\
& e_{22}(i, j)+e_{22}(i+1, j+1)=\varepsilon_{(i-9) / 2,(j-9) / 2}^{4}, \quad i, j \in\{11,13,15,17,19\}, \\
& e_{22}(21,21)+e_{22}(22,22)=\varepsilon_{11}^{5} .
\end{aligned}
$$

These satisfy (2.3) and (2.4) so it follows that

$$
e=\xi_{1}+\xi_{2}+\xi_{6}+\xi_{7}+\xi_{12}
$$


defines an idempotent with $S_{1} e S_{1}=S_{1}$ and (2.1), (2.2), (2.6) and (2.7) become applicable. Thus we can shift our attention to the ring $e S_{1} e$ and the module $X \varepsilon e$, which has a $Z_{(2)}$-generating set comprising $\mathbf{x}_{1}, \mathbf{a}_{1}, \mathbf{a}_{2}, \mathbf{a}_{6}, \mathbf{a}_{7}, \mathbf{a}_{8}, \mathbf{a}_{17}$ and $\mathbf{a}_{18}$ which we rename $x_{1}, w_{1}, \ldots, w_{7}$ respectively in accordance with II. We write $T_{1}$ for the image of $e S_{1} e$ in the algebra End $Z_{Z_{(2)}} X \varepsilon e$. By an argument similar to that used in II we aim to find $T_{1}$-submodules of $X \varepsilon e$, and as in that case we shall construct $T_{1}$ explicitly (as an algebra of $8 \times 8$ matrices).

Let $T_{1}^{\prime}$ be the set of $8 \times 8$ matrices $\alpha \oplus \beta$ satisfying:

(i) $\alpha$ is a $1 \times 1$ matrix and $\beta$ is an element of $T$ with each $\beta(i, j)$ reduced modulo $2^{s} Z_{(2)}$;

(ii) $\alpha(1,1) \equiv \beta(2,2)(\bmod 2)$.

\subsection{THEOREM. $T_{1}=T_{1}^{\prime}$.}

Proof. This follows the pattern of (4.8) with minor modifications. Almost all the generators of $T_{1}$ arise naturally from the corresponding elements of $T$ and their inclusion in $T_{1}^{\prime}$ follows from (4.8). The exceptions have at most two nonzero entries which occur in the $(1,1)$ and $(3,3)$ positions. $T_{1} \leqslant T_{1}^{\prime}$ follows once we have carried out the straightforward check that in each case those entries are congruent modulo 2.

The reverse inclusion follows similarly. $T_{1}^{\prime}$ has 31 generators, 29 of which are in $T_{1}$ by (4.8). The exceptions are $2 e_{8}(3,3)$ which is in $T_{1}$ because it is $\left[2 e_{8}(3,7)-\right.$ $\left.e_{8}(3,8)\right] e_{8}(7,3)$ and $e_{8}(1,1)+e_{8}(3,3)$ which is in $T_{1}$ as the restriction of $\xi_{2}$. This completes the proof of the theorem.

Now let $W_{1}$ be the image of $W$ in XEe. Each submodule of $W_{1}$ can be expressed in terms of generators which are the images of $\mathbf{u}, \ldots, \mathbf{u}_{5}, \mathbf{v}_{4}, \mathbf{v}_{5}$ according to (6.3). As usual we adopt the same symbols for the corresponding elements of $W_{1}$. Let $M$ be a submodule of $X \varepsilon e$ and set $M \cap Z_{(2)} \mathbf{x}_{1}=2^{p} Z_{(2)} \mathbf{x}_{1}, M \cap Z_{(2)} \mathbf{u}_{i}=2^{u(i)} Z_{(2)} \mathbf{u}_{i}$ and $M \cap Z_{(2)} \mathbf{v}_{j}=2^{v(j)} Z_{(2)} \mathbf{v}_{j}$. Let $M \cap W_{1}=M_{1}$. Then since $\left.T_{1}\right|_{W_{1}}$ contains $e_{8}(3,3)$ we can write $M_{1}=\left(M \cap Z_{(2)} \mathbf{u}_{2}\right) \oplus M_{2}$. Also if $N=2^{p} Z_{(2)} \mathbf{x}_{1} \oplus M_{1}$ then since $T_{1}$ contains $e_{8}(1,1)$ we have $2 M \leqslant N$. Finally, since $T_{1}$ contains $e_{8}(1,1)+e_{8}(3,3)$

$$
M=\left[M \cap\left(Z_{(2)} \mathbf{x}_{1} \oplus Z_{(2)} \mathbf{u}_{2}\right)\right] \oplus M_{2}:
$$

The first direct summand is of the same form as those analysed in Section 6 of II; by a similar argument we conclude that $M \cap\left(Z_{(2)} \mathbf{x}_{1} \oplus Z_{(2)} \mathbf{u}_{2}\right)$ is generated by $2^{p} Z_{(2)} \mathbf{x}_{1} \oplus 2^{u(2)} Z_{(2)} \mathbf{u}_{2}$ and $j\left(2^{p-1} \mathbf{x}_{1}+2^{u(2)-1} \mathbf{u}_{2}\right)$ where $j$ is 0 or 1 and if $j=1$ then $p \geqslant 1$ and $u(2) \geqslant 1$. All that remains is to impose the additional conditions that $M$ contain the images of $2^{p} \mathbf{x}_{1}$ and possibly $2^{p-1} \mathbf{x}_{1}+2^{u(2)-1} \mathbf{u}_{2}$ under the 
generators of $T_{1}$ and then make the translation back to the group context. We shall omit the details of this straightforward calculation for the moment preferring instead to subsume them in a more general statement later on.

\section{Subgroups of $F^{\prime}$}

We carry out the same procedure described at the beginning of Section 3 on the abelian section $\mathfrak{B}_{2^{r}}\left(F^{\prime}\right) N_{2}^{2^{s}} N_{3} / N_{2}^{2^{r}} N_{3}^{2^{s}}$ of $F$ writing $Y$ for the corresponding $Z_{(2)} G$-module. Now the only element of $Y$ corresponding to a basic commutator of weight 2 fixed by $\varepsilon$ is $[y, x]^{2^{r}}$ and we denote this element by $\mathbf{y}$. The labels of the other elements of $F$ corresponding to the generators $\mathbf{x}_{1}, \ldots, \mathbf{a}_{18}$ of $X \varepsilon$ are transferred to their images in $Y \varepsilon$. Thus $Y \varepsilon$ is a direct sum of 23 cyclic $Z_{(2)}$-modules, one annihilated by 0 only, four by $2^{r-s} Z_{(2)}$ and 18 by $2^{s} Z_{(2)}$. Also $Y \varepsilon=Y \varepsilon_{2}$ $\oplus Y \varepsilon_{3} \oplus Y \varepsilon_{4}$ and these are generated by $\left\{\mathbf{y}, \mathbf{x}_{1}, \mathbf{x}_{2}, \mathbf{a}_{1}, \mathbf{a}_{2}, \mathbf{a}_{3}\right\},\left\{\mathbf{x}_{3}, \mathbf{x}_{4}, \mathbf{a}_{4}, \ldots, \mathbf{a}_{12}\right\}$ and $\left\{\mathbf{a}_{13}, \ldots, \mathbf{a}_{18}\right\}$ respectively. With reference to the generating set $\mathbf{y}, \mathbf{x}_{1}, \ldots, \mathbf{x}_{4}$, $\mathbf{a}_{1}, \ldots, \mathbf{a}_{18}$ End $_{Z_{(2)}} Y \varepsilon$ can be identified with a homomorphic image of $\operatorname{Mat}\left(23, Z_{(2)}\right)$ (Jacobson (1979)). Denote by $S_{2}$ the image of $\varepsilon Z_{(2)} G \varepsilon$ in End $Z_{(2)} Y \varepsilon$. (Here the matrix algebra representing End $Z_{(2)} Y \varepsilon$ is more complicated: there are two cases according to whether $r-s \leqslant s$ or $r-s>s$. The former would have the generators in their present order; the latter would interchange the $\mathbf{a}_{i}$ and the $\mathbf{x}_{i}$. However this distinction makes no significant difference for the algebra $S_{2}$ so we can safely ignore it.)

Each entry apart from the first in the first column of each matrix in $\operatorname{End}_{Z_{12}} Y \varepsilon$ is zero. Moreover by virtue of 2.7 each entry apart from the first in the first row of each matrix in $S_{2}$ is zero with the possible exception of those occurring in the columns corresponding to $\mathbf{a}_{8}, \mathbf{a}_{10}$ and $\mathbf{a}_{12}$ : the entries in these columns are from $2^{r-1} Z_{(2)}$. The lower right $22 \times 22$ submatrix of each matrix in $S_{2}$ can be obtained from the corresponding matrix in $S_{1}$ simply by reading its $4 \times 4$ direct summand modulo $2^{r-s} Z_{(2)}$. Thus it remains to describe the action of the generators of $S_{2}$ (obtained from (3.4)) on the single element $\mathbf{y}$.

\subsection{Action on $Y \varepsilon_{2}$.}

$$
\begin{aligned}
\mathbf{y} \varepsilon_{s} \sigma_{2} \varepsilon_{2} & =-\mathbf{y}, \quad \mathbf{y} \varepsilon_{2} \mu_{\kappa} \varepsilon_{2}=\kappa \mathbf{y} . \\
\mathbf{y} \varepsilon_{2} \tau \varepsilon_{2} & =\mathbf{y}, \quad \mathbf{y}(2 \rightarrow 3)=2^{r-1}\left(2^{r}-1\right) \mathbf{a}_{12} .
\end{aligned}
$$

The action of the elements $\xi_{1}, \ldots, \hat{\xi}_{4}, \hat{\xi}_{5}, \ldots, \xi_{12}$ (of $S_{2}$ !) is checked by direct calculation: they all annihilate y except $\xi_{1}$ which acts on $Y \varepsilon_{2}$ as $\operatorname{diag}(1,0,0,1,0,0)$. Hence they are pairwise orthogonal idempotents with sum 1 in $S_{2}$, $\xi_{1}$ is just 
$e_{23}(1,1)+e_{23}(6,6)$ and so translating 3.5 to the present context we have

4.2 Lemma. The following elements are in $S_{2}$ :

$$
\begin{array}{ll}
e_{23}(1,1)+e_{23}(6,6), & \\
e_{23}(i, j)+e_{23}(i+5, j+5), & i, j \in\{2,3,4,5\}, \\
e_{23}(i, j)+e_{23}(i+1, j+1), & i, j \in\{12,14,16,18,20\} .
\end{array}
$$

After relabelling these elements as before we can put $e=\xi_{1}+\xi_{2}+\xi_{6}+\xi_{7}+$ $\xi_{12}$ so that $S_{2} e S_{2}=S_{2}$. Let $T_{2}$ stand for the image of $S_{2}$ in the algebra End $Z_{(2)} Y \varepsilon e$ identified with an algebra of matrices in the usual way. Let $T_{2}^{\prime}$ be the set of $9 \times 9$ matrices satisfying conditions:

(i) the first row is $(\gamma 00000 \delta 00)$ with $\gamma \in Z_{(2)}$ and $\delta \in 2^{r-1} Z_{(2)}$;

(ii) the first column entries apart from the first are zero;

(iii) the lower right $8 \times 8$ submatrix is an element $\alpha \oplus \beta$ of $T_{1}$ with $\alpha$ reduced modulo $2^{r-s} Z_{(2)}$;

(iv) $\gamma \equiv \beta(1,1)(\bmod 2)$.

Then we have the following theorem whose proof is after the pattern of 3.6 and (4.8) and will be omitted.

\subsection{ThEOREM. $T_{2}=T_{2}^{\prime}$.}

The analysis of submodules of $Y \varepsilon e$ is analogous to that of submodules of $X \varepsilon e$ carried out in Section 3. Write $W_{2}$ for the image of $W$ in $Y \varepsilon e$. Let $M$ be a submodule of $Y_{\varepsilon e}$ and with obvious adaptations of the notation set

$$
\begin{aligned}
& M \cap Z_{(2)} \mathbf{y}=2^{p^{(1)}} Z_{(2)} \mathbf{y}, \\
& M \cap Z_{(2)} \mathbf{x}_{1}=2^{p^{(2)}} Z_{(2)} \mathbf{x}_{1}, \\
& M \cap W_{2}=M_{1}, \\
& M_{1}=\left(M \cap Z_{(2)} \mathbf{u}_{1}\right) \oplus\left(M \cap Z_{(2)} \mathbf{u}_{2}\right) \oplus M_{2}
\end{aligned}
$$

(using the fact that $e_{9}(3,3)$ and $e_{9}(4,4)$ are in $\left.T_{2}\right|_{w_{2}}$ ).

These lead to

$$
M=\left[M \cap\left(Z_{(2)} \mathbf{y} \oplus Z_{(2)} \mathbf{u}_{1}\right)\right] \oplus\left[M \cap\left(Z_{(2)} \mathbf{x}_{1} \oplus Z_{(2)} \mathbf{u}_{2}\right)\right] \oplus M_{2} .
$$

The first direct summand is generated by $2^{p(1)} Z_{(2)} \mathbf{y} \oplus 2^{u(1)} Z_{(2)} \mathbf{u}_{1}$ and $i\left(2^{p(1)-1} \mathbf{y}+\right.$ $\left.2^{u(1)-1} \mathbf{u}_{1}\right)$ where $i=0$ or 1 and if $i=1$ then $p(1) \geqslant 1$ and $u(1) \geqslant 1$.

Now $\left[M \cap\left(Z_{(2)} \mathbf{x}_{1} \oplus Z_{(2)} \mathbf{u}_{2}\right)\right] \oplus M_{2}$ can be completely described in terms of the known (but as yet unstated) parametrization of the fully invariant subgroups of $F$ contained in $N_{2}$. Again we need only impose the conditions that $M$ admit $T_{2}$ and translate back to $F$, and once again details are left until later. 


\section{The final result}

Different methods are needed to cope with 2 '-isolated subgroups $H$ of $F$ which are not contained in $F^{\prime}$. The starting point here is that each such $H$ must be of the form $\left(F^{\prime} \cap H\right) \mathfrak{B}_{2^{q}}(F)$ for some nonnegative integer $q$, which is identified by taking $2^{q}$ to be the exponent of $F / H$. This is virtually a paraphrase of $\mathrm{B}$. $\mathrm{H}$. Neumann's classical result (12.12 in Hanna Neumann (1967)) that each law is equivalent to an exponent law and a commutator law. Of course $F^{\prime} \cap H \geqslant F^{\prime} \cap$ $\mathfrak{B}_{2^{q}}(F)$; conversely, if $H_{1}$ is any $2^{\prime}$-isolated fully invariant subgroup of $F$ between $F^{\prime} \cap \mathfrak{B}_{2^{q}}(F)$ and $F^{\prime}$, then for $H$ defined by $H=H_{1} \mathfrak{B}_{2^{q}}(F)$ one has that the exponent of $F / H$ is $2^{q}$ and $F^{\prime} \cap H=H_{1}$. Thus the general problem is reduced to identifying the $F^{\prime} \cap \mathfrak{B}_{2^{q}}(F)$ in terms of the (yet to be stated) parametrization of the $2^{\prime}$-isolated fully invariant subgroups of $F$ in $F^{\prime}$. In those terms, one can then recognise the $H_{1}$ which lie between $F^{\prime} \cap \mathfrak{B}_{2^{q}}(F)$ and $F^{\prime}$.

We can ignore the cases $q=0$ and $q=1$, for then $\mathfrak{B}_{2^{q}}(f) \geqslant F^{\prime}$; so henceforth $q \geqslant 2$. Our aim here is to prove that $F^{\prime} \cap \mathfrak{B}_{2^{q}}(F)$ is the fully invariant closure of $[y, x]^{2^{q-1}} \mathbf{u}_{1}^{2^{4-2}}$ and $\mathfrak{B}_{2^{q}}\left(F^{\prime}\right) N_{2}^{2^{4-1}}$. This is good enough to enable one to complete the work; the subsequent details follow the pattern we have already established, and instead of elaborating them we proceed directly to the statement of the main result.

For simplicity, write $F^{\prime} \cap \mathfrak{B}_{2^{q}}(F)$ as $D$.

The first step is to quote again from the classification of varieties of nilpotent groups of class at most 3:

$$
D N_{3}=\Re_{2^{q-1}}\left(F^{\prime}\right) N_{2}^{2^{q-1}} N_{3} .
$$

(Strictly speaking, the left hand side has to be rewritten as $F^{\prime} \cap \mathfrak{B}_{2^{q}}(F) N_{3}$, using the modular law, before we are entitled to quote.) By $2.3, D \geqslant N^{2^{4-1}}$; as obviously $D \geqslant \mathfrak{B}_{2^{q}}\left(F^{\prime}\right)$, we already have that

$$
D \geqslant \mathfrak{B}_{2^{q}}\left(F^{\prime}\right) N_{2}^{2^{q-1}} .
$$

We shall use repeatedly the following fact.

5.3 LEMMA. If $u$ and $v$ are elements of a nilpotent group $C$ of class at most 3 , then $u^{2^{q}} v^{2^{q}} \equiv(u v)^{2^{q}}$ modulo $\left(C^{\prime}\right)^{2^{q-1}}$.

The proof is a straightforward collection, a special case of Lemma 5.7 below so we omit it. For the first application, we take $C$ as the subgroup of $F$ generated by $x$ and $[x, y]$ and note that now $C^{\prime} \leqslant N_{2}$, so we may conclude that

$$
\left[x^{2^{q}}, y\right]=x^{-2^{q}}(x[x, y])^{2^{q}} \equiv[x, y]^{2^{q}} \bmod N_{2}^{2^{q-1}},
$$


and hence $\left[x^{2^{4}}, y\right] \in \mathcal{B}_{2^{q}}\left(F^{\prime}\right) N_{2}^{2^{q-1}}$. This observation proves

5.4 LeMma. $\mathfrak{B}_{2^{q}}(F) / \mathfrak{B}_{2^{q}}\left(F^{\prime}\right) N_{2}^{2^{q-1}}$ is a central section of $F$.

For the second application of 5.3, let $a$ be any element of $F$ and $\varphi, \psi$ two endomorphisms of $F$ which agree on $F / F^{\prime}$, so $a \varphi=(a \psi) b$ for some element $b$ of $F^{\prime}$. Take $C$ as the subgroup generated by $a \psi$ and $b$, noting that again $C^{\prime} \leqslant N_{2}$. Now 5.3 yields that

$$
a^{2^{4}} \varphi=(a \varphi)^{2^{q}}=((a \psi) b)^{2^{q}}=\left(a^{2^{q}} \psi\right) b^{2^{q}} \bmod N_{2}^{2^{q-1}},
$$

so $\varphi$ and $\psi$ agree on the element $a^{2^{q}} \mathfrak{B}_{2^{q}}\left(F^{\prime}\right) N_{2}^{2^{q-1}}$ of the section considered in 5.4. That section is generated by such elements (as group, not only as fully invariant subgroup), so we may conclude

5.5 LeMMA. If two endomorphisms of $F$ agree on $F / F^{\prime}$, they also agree on $\mathfrak{B}_{2^{q}}(F) / \mathfrak{B}_{2^{q}}\left(F^{\prime}\right) N_{2}^{2^{q-1}}$.

Let $d$ be the element of $D$ defined by

$$
(x y)^{2^{q}}=x^{2^{q}} y^{2^{q}} d,
$$

and let $D_{1}$ be the fully invariant closure of $d$ and $\mathfrak{B}_{2^{q}}\left(F^{\prime}\right) N_{2}^{2^{q-1}}$ in $F$. The next step is to prove

5.6 Lemma. $D=D_{1}$.

Proof. Clearly, $D \geqslant D_{1}$. We know that $\mathfrak{B}_{2^{q}}(F) / D \simeq \mathfrak{B}_{2^{q}}(F) F^{\prime} / F^{\prime}=$ $\mathfrak{B}_{2^{q}}\left(F / F^{\prime}\right)$, so $\mathfrak{B}_{2^{q}}(F) / D$ is free abelian of rank 4 , and therefore it cannot be written as a proper homomorphic image of any 4-generator abelian group. Thus the lemma will follow if we can establish that $B_{2^{4}}(F) / D_{1}$ is a 4-generator abelian group. We already know from 5.4 that it is abelian; we shall now show that it is generated by $x^{29} D_{1}, \ldots, t^{29} D_{1}$. To this end, it is clearly sufficient to show that the subgroup of $\mathfrak{B}_{2^{4}}(F) / D_{1}$ generated by these elements admits all endomorphisms of $F$. This subgroup obviously admits all endomorphisms which merely permute or power the generators $x, y, z, t$. It also admits the endomorphism which maps $x$ to $x y$ and leaves $y, z, t$ fixed: for, this will map $x^{2^{4}} D_{1}$ to $(x y)^{29} D_{1}$ which is equal to $\left(x^{2^{q}} D_{1}\right)\left(y^{2^{q}} D_{1}\right)$ by the definition of $D_{1}$. We know from (3.1) that each endomorphism of $F$ will agree on $F / F^{\prime}$ with some composite of the endomorphisms just considered; hence it follows by 5.5 that our subgroup will admit it. This proves the lemma. 
To reach our stated aim, it remains to prove that

$$
d \equiv[y, x]^{2^{q-1}} \mathbf{u}_{1}^{2^{q-2}} \text { modulo } B_{2^{4}}\left(F^{\prime}\right) N_{2}^{2^{q-1}} .
$$

At this point, one cannot avoid a complicated collection to find out just what $d$ is.

\subsection{Lemma.}

$$
(x y)^{2^{q}}=x^{2^{q}} y^{2^{q}}[y, x]^{\alpha}[y, x, x]^{\beta}[x, y, y]^{\gamma}[y, x, x, x]^{\delta} u_{1}^{\varepsilon}
$$

where

$$
\begin{aligned}
& \alpha=\left(\begin{array}{c}
2^{q} \\
2
\end{array}\right) \equiv 2^{q-1} \quad\left(\bmod 2^{q}\right) \\
& \beta=\left(\begin{array}{c}
2^{q} \\
3
\end{array}\right) \equiv 0 \quad\left(\bmod 2^{q-1}\right) \\
& \gamma=-\left(\begin{array}{c}
2^{q} \\
3
\end{array}\right)-\left(\begin{array}{c}
2^{q}+1 \\
3
\end{array}\right) \equiv 0 \quad\left(\bmod 2^{q-1}\right) \\
& \delta=-2\left(\begin{array}{c}
2^{q}+1 \\
4
\end{array}\right) \equiv 0 \quad\left(\bmod 2^{q-1}\right), \\
& \varepsilon=\left(\begin{array}{c}
2^{q} \\
4
\end{array}\right)+2\left(\begin{array}{c}
2^{q}+1 \\
4
\end{array}\right) \equiv 2^{q-2} \quad\left(\bmod 2^{q-1}\right)
\end{aligned}
$$

This confirms the claim concerning $d$; the straightforward but tedious proof is omitted, as in the remaining detail which leads to our main result.

We recall the convention $2^{\infty}=0$, and supplement it by the usual $\infty \pm 1=\infty$. It will be convenient to have the following shorthand available: $\left(u^{\prime}(1), \ldots, v^{\prime}(5)\right.$; $\left.k^{\prime}, l^{\prime}, m^{\prime}, n^{\prime}\right) \leqslant M$ will mean that $u^{\prime}(1), \ldots, n^{\prime}$ satisfy the conditions (7), (8), (9) and (10) of (6.2); when $s$ is a nonnegative integer, we write $2^{s} W$ for $(s, \ldots, s ; 0,0,1,1)$.

5.8 THEOREM. The 2'-isolated fully invariant subgroups of $F$ are in one-to-one correspondence with the ordered 16-tuples

$$
(q ; r ; s ; u(1), \ldots, u(5), v(4), v(5) ; i ; j ; k, l, m, n)
$$

which satisfy conditions (1), (2) of (6.1), (4), (5), (6) of (6.2), (11), (12), (13), (14) of (6.3) and the following.

(15) $q, r, s$ are nonnegative integers or $\infty$, while $i, j \in\{0,1\}$.

(16) If $q \leqslant 1$ then $r=0$.

(17) If $r=0$ then the 16-tuple is

$$
(q ; 0 ; 0 ; 0, \ldots, 0 ; 0 ; 0 ; 0,0,1,1) .
$$

(18) If $q \geqslant 2$ and $r \geqslant 1$ then $s \leqslant q-1, s \leqslant r$, either $s=\infty$ or $2^{s} W \leqslant M$, and either $r \leqslant q-1$ and $u(1) \leqslant q-2$ and $u(4) \leqslant r-1$, or $r=q$ and $u(1)=q-1$ and $i=1$. 
(19) If $i=1$ then $1 \leqslant u(1) \leqslant s<r<\infty$ and

$(u(1), u(1), u(1)-1, u(1), u(1)-1, r-1, r-1 ; 1,0,0,0) \leqslant M$.

(20) If $j=1$ then $1 \leqslant u(2)<s<\infty, 2^{s-1} W \leqslant M$ and

$$
u(2) \geqslant \max \{u(1)-1, u(3), u(4), u(5)+1\} .
$$

The corresponding subgroup of $F$ is the fully invariant closure of

$$
\begin{gathered}
x^{2^{4}}, \quad[y, x]^{2^{r}}, \quad[y, x, x]^{2^{s}}, \\
\left([y, x]^{2^{r-1}} \mathbf{u}_{2}^{2^{u(1)-1}}\right)^{i}, \quad\left([y, x, x]^{2^{s-1}} \mathbf{u}_{2}^{2^{u(2)-1}}\right)^{j},
\end{gathered}
$$

and the elements listed in (7.1). It contains the subgroup corresponding to $\left(q^{\prime} ; \ldots, n^{\prime}\right)$ if and only if $q \leqslant q^{\prime}, r \leqslant r^{\prime}, s \leqslant s^{\prime},\left(u^{\prime}(1), \ldots, n^{\prime}\right) \leqslant M$, and

(21) if $i^{\prime}=1$ then either $r \leqslant r^{\prime}-1$ and $u(1) \leqslant u^{\prime}(1)-1$ or $r=r^{\prime}$ and $u(1)=$ $u^{\prime}(1)$ and $i=1$;

(22) if $j^{\prime}=1$ then either $s \leqslant s^{\prime}-1$ and $u(2) \leqslant u^{\prime}(2)-1$ or $s=s^{\prime}$ and $u(2)=$ $u^{\prime}(2)$ and $j=1$.

It follows, in particular, that the subgroup $\mathfrak{B}_{4}(F)$ corresponds to

$$
(2 ; 2 ; 1 ; 1,1,0,1,0,1,0 ; 1 ; 0 ; 0,0,1,0) \text {. }
$$

This has been compared and found to agree with the known structure of the Burnside group $B(4,4)$ (see, for instance, Hall (1973)). The isolated fully invariant subgroups are readily identified as those with all parameters in $\{0, \infty\}$ except that when the middle group of seven parameters consists of zeros the last two parameters are ones. There is no problem in identifying the parameters of the isolator from the parameters of a subgroup, or in obtaining at least a crude upper estimate for the exponent of their quotient (if $p$ is the sum of the finite parameters of the subgroup, $2^{2+p}$ will always do). Thus the reduction obtained in I does in fact apply. It is implicit in our arguments that, given any subgroup by its parameters and any element of $F$, one can decide whether the element belongs to the subgroup, but to make this explicit and elaborate an algorithm is beyond the scope of this work. We have also found algorithms for calculating from the parameters of two subgroups the parameters of their intersection and product. These are very easy to state and apply but to prove that they work involves a long and complicated case by case analysis so we do not include them.

Since each variety of nilpoint groups of class at most 4 is defined by its 4-variable laws (see 34.15 and 34.34 in Hanna Neumann (1967)), the result we have reached is equivalent to determining all 2 '-torsionfree varieties of nilpotent groups of class at most 4 . In view of $I$, this completes the task of finding all varieties of nilpotent groups of class at most 4 . 


\section{References}

Patrick Fitz atrick and L. G. Kovács, 'Varieties of nilpotent groups of class four (I)', J. A ustral. Math. Soc., Ser. A 35 (1983), 59-73.

Patrick Fitzpatrick, 'Varieties of nilpotent groups of class four (II)', J. Austral. Math. Soc., Ser. A 35 (1983), 74-108.

N. Jacobson, Basic algebra I (Freeman, 1974).

B. Jónsson, 'Varieties of groups of nilpotency three', Notices Amer. Math. Soc. 13 (1966), 488.

H. Neumann, Varieties of groups (Ergebnisse der Mathematik und ihrer Grenzgebiete, 37. SpringerVerlag, Berlin, Heidelberg, New York, 1967).

Department of Mathematics

University College

Cork

Ireland 